PROCEEDINGS OF THE

AMERICAN MATHEMATICAL SOCIETY

Volume 137, Number 2, February 2009, Pages 425-429

S 0002-9939(08)09688-3

Article electronically published on September 15, 2008

\title{
GENERALIZING A THEOREM OF P. HALL ON FINITE-BY-NILPOTENT GROUPS
}

\author{
GUSTAVO A. FERNÁNDEZ-ALCOBER AND MARTA MORIGI
}

(Communicated by Jonathan I. Hall)

\begin{abstract}
Let $\gamma_{i}(G)$ and $Z_{i}(G)$ denote the $i$-th terms of the lower and upper central series of a group $G$, respectively. In 1956 P. Hall showed that if $\gamma_{i+1}(G)$ is finite, then the index $\left|G: Z_{2 i}(G)\right|$ is finite. We prove that the same result holds under the weaker hypothesis that $\left|\gamma_{i+1}(G): \gamma_{i+1}(G) \cap Z_{i}(G)\right|$ is finite.
\end{abstract}

\section{INTRODUCTION}

If $G$ is an arbitrary group, a classical theorem of Schur shows that if the center $Z(G)$ has finite index in $G$, then the derived subgroup $G^{\prime}$ is finite. More precisely, if $|G: Z(G)|=n$, then $\left|G^{\prime}\right| \leq f(n)$ for some function $f$ (see [6, page 102] for an explicit description of $f$ ). Schur's theorem was later generalized by Baer (see 5 , 4.5.1]) to other terms of the upper/lower central series; namely, he proved that if $\left|G: Z_{i}(G)\right|$ is finite, then $\gamma_{i+1}(G)$ is also finite. The converse does not hold in general; however in 2], P. Hall obtained that if $\gamma_{i+1}(G)$ is finite, then $\left|G: Z_{2 i}(G)\right|$ is finite, thus proving that finite-by-nilpotent groups are nilpotent-by-finite. Note that both Baer's and Hall's results can be stated quantitatively. (See the remark after Theorem 2.2 below and [6, page 118], respectively.) A stronger form of Hall's result is known for the case $i=1$ : it suffices to assume that $\left|G^{\prime}: G^{\prime} \cap Z(G)\right|$ is finite in order to conclude the finiteness of $\left|G: Z_{2}(G)\right|$. This result was obtained (in quantitative form) by Isaacs in 3 , when $G$ is finite, and then independently by Fernández-Alcober and Moretó [1, Theorem E], and by Podoski and Szegedy [4, Theorem 1] when $G$ is an arbitrary group. The goal of this paper is to prove the following generalization of this last property to an arbitrary value of $i$.

Theorem A. Let $G$ be a group such that $n=\left|\gamma_{i+1}(G): \gamma_{i+1}(G) \cap Z_{i}(G)\right|$ is finite. Then $\left|G: Z_{2 i}(G)\right|$ is finite and can be bounded by a function of $n$.

We have made no attempt at giving a sharp bound for $\left|G: Z_{2 i}(G)\right|$ in terms of $n$ in Theorem A. In the case $i=1$, a bound which is essentially best possible can be found in 4 .

Related to Theorem A, the following two questions arise naturally:

Received by the editors November 7, 2007.

2000 Mathematics Subject Classification. Primary 20F14.

Key words and phrases. Upper and lower central series; Finite-by-nilpotent groups.

The first author is supported by the Spanish Ministry of Science and Education, grant MTM2004-04665, partly with FEDER funds, and by the Basque Government, grant IT-252-07.

The second author is partially supported by MIUR (Project "Teoria dei Gruppi e applicazioni") and thanks the University of the Basque Country for its hospitality. 
(1) To what extent is Theorem A best possible? If the weaker condition that $\left|\gamma_{i+1}(G): \gamma_{i+1}(G) \cap Z_{i+1}(G)\right|$ is finite holds, can we conclude that $\mid G$ : $Z_{2 i}(G) \mid$ is finite?

(2) In the case that $\gamma_{i+1}(G)$ is finite, if $G$ is also finitely generated, then a stronger result holds, namely $\left|G: Z_{i}(G)\right|$ is finite. Is this true also under the hypothesis of Theorem A? Does it follow at least that $\left|G: Z_{j}(G)\right|$ is finite for some $j$ smaller than $2 i$ ?

The answer to both these questions is negative. To see this, for arbitrary $c$, consider a finitely generated nilpotent group $G$ of class $c$ in which the upper and lower central series coincide and such that $\left|G: Z_{c-1}(G)\right|$ is infinite. For example, one can take the semidirect product $G=B \ltimes A$, where $B=\langle b\rangle$ is an infinite cyclic group, $A$ is the free abelian group on free generators $a_{1}, \ldots, a_{c}$, and $b$ acts on $A$ by $a_{i}^{b}=a_{i} a_{i+1}$ for $1 \leq i \leq c-1$ and $a_{c}^{b}=a_{c}$. Now if $i \geq 1$ is any fixed integer, we get counterexamples to the first and the second questions by choosing $c=2 i+1$ and $c=2 i$, respectively.

Finally, we observe that combining our result with Baer's theorem it follows that if $\left|\gamma_{i+1}(G): \gamma_{i+1}(G) \cap Z_{i}(G)\right|$ is finite, then $\gamma_{2 i+1}(G)$ is also finite. Actually, one of the key arguments in our proof of Theorem $\mathrm{A}$ is the following generalization of this fact, which might be interesting in its own right.

Theorem B. Let $G$ be a group such that $\left|\gamma_{s}(G): \gamma_{s}(G) \cap Z_{t}(G)\right|$ is finite for some $s, t$. Then $\left|\gamma_{s+j}(G): \gamma_{s+j}(G) \cap Z_{t-j}(G)\right|$ is finite for every $j$ such that $0 \leq j \leq t$. In particular, $\gamma_{s+t}(G)$ is finite.

\section{The Results}

The notation we use is standard. Moreover, following the book [5], if $A$ and $B$ are subgroups of a group $G$ and $n$ is a natural number, we define recursively:

$$
\left[A,{ }_{0} B\right]=A, \quad\left[A,{ }_{n} B\right]=\left[A,{ }_{n-1} B, B\right] .
$$

Throughout the paper, we will repeatedly use the following well-known result (see for instance 5.1.10 in [5]).

Three Subgroup Lemma. Let $H, K, L$ be subgroups of a group $G$. If two of the commutator subgroups $[H, K, L],[K, L, H],[L, H, K]$ are contained in a normal subgroup of $G$, then so is the third.

Another result which will be used often in our proofs is stated for convenience in the following lemma, whose proof is elementary. Most of the time, we will apply it modulo a normal subgroup.

Lemma 2.1. Let $H, K$ be subgroups of a group $G$. If $[H, K]$ is finite and $H$ is finitely generated, then the centralizer $C_{K}(H)$ has finite index in $K$. More precisely, if $[H, K]$ has order $n$ and $H$ is m-generated, then $\left|K: C_{K}(H)\right| \leq n^{m}$.

We will also need the following result of Baer. The quantitative version stated below follows easily from the proof of 14.5.2 in [5].

Theorem 2.2. Let $M \leq H$ and $N \leq K$ be normal subgroups of a group $G$ such that $|H: M|=h$ and $|K: N|=k$ are finite and $[H, N]=1=[K, M]$. Then $[H, K]$ is finite and its order is at most $h k f(h k)^{2}$, where $f$ is the function arising in the quantitative form of Schur's theorem. 
As a consequence of this result, one can easily obtain Baer's theorem stating that $\gamma_{i+1}(G)$ is finite if $\left|G: Z_{i}(G)\right|$ is. (See [5, 14.5.1].) It follows that the order of $\gamma_{i+1}(G)$ can be bounded by a function of $\left|G: Z_{i}(G)\right|$, as mentioned in the introduction.

The key step in the proof of our main theorem is in the following proposition.

Proposition 2.3. Let $G$ be a group and let $s \geq 1$ be an integer such that $\mid \gamma_{s}(G)$ : $\gamma_{s}(G) \cap Z(G) \mid$ is finite. Then $C_{G}\left(\gamma_{s}(G)\right)$ has finite index in $G$ and $\gamma_{s+1}(G)$ is finite.

Proof. Let $Z=\gamma_{s}(G) \cap Z(G)$. As $\gamma_{s}(G) / Z$ is finite, there exists a finitely generated subgroup $U$ of $G$ such that $\gamma_{s}(G)=\gamma_{s}(U) Z$. By applying P. Hall's theorem to the quotient group $G / Z(G)$, we obtain that $\left|G: Z_{2 s-1}(G)\right|$ is finite. By the theorem of Baer mentioned in the introduction, it follows that $\gamma_{2 s}(G)$ is finite. Since $\gamma_{k}(U) / \gamma_{k+1}(U)$ is finitely generated for every $k=1, \ldots, 2 s-1$, we conclude that all terms of the lower central series of $U$ are finitely generated.

We are going to prove that, for every $j=1, \ldots, s$, there exists a subgroup $H_{j}$ of finite index in $\gamma_{j}(G)$ such that $\left[H_{j}, \gamma_{s-j+1}(U)\right]=1$. Then $\left[H_{1}, \gamma_{s}(G)\right]=$ $\left[H_{1}, \gamma_{s}(U) Z\right]=\left[H_{1}, \gamma_{s}(U)\right]=1$, which proves that $\left|G: C_{G}\left(\gamma_{s}(G)\right)\right|$ is finite.

We prove the existence of $H_{j}$ by reverse induction on $j$. For $j=s$, we take $H_{s}=Z$. Suppose now that we already have $H_{j+1}$ of finite index in $\gamma_{j+1}(G)$ such that $\left[H_{j+1}, \gamma_{s-j}(U)\right]=1$, and let us see how to construct the subgroup $H_{j}$. Let $K_{j}=C_{\gamma_{j}(G)}\left(\gamma_{s-j}(U) Z / Z\right)$. Since $\gamma_{s-j}(U) Z / Z$ is finitely generated and $\left[\gamma_{j}(G), \gamma_{s-j}(U)\right] Z / Z \leq \gamma_{s}(G) / Z$ is finite, it follows from Lemma 2.1 that $K_{j}$ has finite index in $\gamma_{j}(G)$. Also

$$
\left[K_{j}, \gamma_{s-j}(U), U\right] \leq[Z, U]=1 .
$$

Let now $D_{j+1}=C_{\gamma_{j+1}(G)}\left(\gamma_{s-j}(U)\right)$. Since $\left[H_{j+1}, \gamma_{s-j}(U)\right]=1$, we have $H_{j+1} \leq$ $D_{j+1}$ and consequently $\left|\gamma_{j+1}(G): D_{j+1}\right|$ is finite. Consider $T_{j}=\gamma_{j}(G) U$. We claim that $D_{j+1}$ is normal in $T_{j}$. On the one hand, clearly $U$ normalizes $D_{j+1}$. On the other hand, we have $\left[\gamma_{j}(G), \gamma_{s-j}(U), D_{j+1}\right] \leq\left[\gamma_{s}(G), D_{j+1}\right]=\left[\gamma_{s}(U) Z, D_{j+1}\right]=1$ and $\left[\gamma_{s-j}(U), D_{j+1}, \gamma_{j}(G)\right]=1$ by the definition of $D_{j+1}$. By the Three Subgroup Lemma, $\left[D_{j+1}, \gamma_{j}(G), \gamma_{s-j}(U)\right]=1$ and consequently also $\gamma_{j}(G)$ normalizes $D_{j+1}$.

Now we work in the quotient group $T_{j} / D_{j+1}$. Since $\left[\gamma_{j}(G), U\right] D_{j+1} / D_{j+1} \leq$ $\gamma_{j+1}(G) / D_{j+1}$ is finite and $U$ is finitely generated, it follows that the centralizer $L_{j}$ of $U D_{j+1} / D_{j+1}$ in $\gamma_{j}(G)$ has finite index in $\gamma_{j}(G)$. Observe that

$$
\left[L_{j}, U, \gamma_{s-j}(U)\right] \leq\left[D_{j+1}, \gamma_{s-j}(U)\right]=1
$$

Finally, let $H_{j}=K_{j} \cap L_{j}$. Then $\left|\gamma_{j}(G): H_{j}\right|$ is finite. Moreover, using (11) and (2) and the Three Subgroup Lemma we obtain that $\left[\gamma_{s-j}(U), U, H_{j}\right]=1$, that is, $\left[\gamma_{s-j+1}(U), H_{j}\right]=1$, as desired.

Now in order to prove that $\gamma_{s+1}(G)$ is finite we apply Theorem 2.2 with $M=$ $C_{G}\left(\gamma_{s}(G)\right), H=G, N=\gamma_{s}(G) \cap Z(G)$ and $K=\gamma_{s}(G)$. It follows that $\left[G, \gamma_{s}(G)\right]=$ $\gamma_{s+1}(G)$ is finite.

Let us remark that if $N$ is a normal subgroup of a group $G$ and $|N: N \cap Z(G)|$ is finite, it does not follow that $\left|G: C_{G}(N)\right|$ or $[N, G]$ is finite. For example, let $p$ be a prime and let $H$ and $N$ be two elementary abelian $p$-groups with countable bases $\left\{x_{i}\right\}_{i \geq 1}$ and $\left\{y_{j}\right\}_{j \geq 0}$, respectively. We define an action of $H$ on $N$ so that $x_{i}$ centralizes all $y_{j}$ with $j \geq 1$ and $y_{0}^{x_{i}}=y_{0} y_{i}$. Then in the semidirect product 
$G=H \ltimes N$ we have $|N: N \cap Z(G)|=p$, but both $\left|G: C_{G}(N)\right|$ and $[N, G]$ are infinite.

Corollary 2.4. Let $G$ be a group such that $\left|\gamma_{s}(G): \gamma_{s}(G) \cap Z_{t}(G)\right|$ is finite for some $s, t$. Then $\left|\gamma_{s+j}(G): \gamma_{s+j}(G) \cap Z_{t-j}(G)\right|$ is finite for every $j$ such that $0 \leq j \leq t$. In particular, $\gamma_{s+t}(G)$ is finite.

Proof. By induction on $t$ it suffices to prove that $\left|\gamma_{s+1}(G): \gamma_{s+1}(G) \cap Z_{t-1}(G)\right|$ is finite. This follows immediately by applying Proposition 2.3 to the quotient group $G / Z_{t-1}(G)$.

The last part of the proof of Theorem A is inspired from Hall's ideas. The main role will be played by a subgroup $C$ with the two properties that $C$ has finite index in $G$ and $\left[C{ }_{s-1} G, C\right] \leq Z_{2 i-s}(G)$ for every $s \geq 1$, with the convention that $Z_{j}(G)=1$ for $j \leq 0$. The following technical lemma will ensure that $C$ has the second property.

Lemma 2.5. Let $G$ be a group and let $C_{j}$ be the centralizer in $G$ of $\gamma_{i+j}(G) / \gamma_{i+j}(G)$ $\cap Z_{i-j}(G)$ for $j=1, \ldots, i$. Put $C=\bigcap_{j=1}^{i} C_{j}$; then $\left[C,{ }_{s-1} G, C\right] \leq Z_{2 i-s}(G)$ for every $s \geq 1$.

Proof. Observe that $C$ is normal in $G$ and so is $\left[C,{ }_{k} G\right]$ for every $k$. We first prove by induction on $k$ that

$$
\left[\left[C,{ }_{k} G\right], \gamma_{\ell}(G)\right] \leq Z_{2 i-k-\ell}(G) \text { for all } k \geq 0 \text { and for all } \ell \geq i+1 .
$$

By definition of $C$, we have $\left[C, \gamma_{\ell}(G)\right] \leq Z_{2 i-\ell}(G)$ for all $\ell \geq i+1$ and this settles the case $k=0$. Assume now that the statement is true for $k$. We have $\left[\gamma_{\ell}(G),\left[C,{ }_{k} G\right], G\right] \leq\left[Z_{2 i-k-\ell}(G), G\right] \leq Z_{2 i-k-\ell-1}(G)$. Also, $\left[G, \gamma_{\ell}(G),\left[C,{ }_{k} G\right]\right]=$ $\left[\gamma_{\ell+1}(G),\left[C,{ }_{k} G\right]\right] \leq Z_{2 i-k-\ell-1}(G)$. So by the Three Subgroup Lemma, it follows that

$$
\left[\left[C,{ }_{k+1} G\right], \gamma_{\ell}(G)\right]=\left[\left[C,{ }_{k} G\right], G, \gamma_{\ell}(G)\right] \leq Z_{2 i-k-\ell-1}(G),
$$

which proves the statement for $k+1$.

Now, in order to prove the lemma, we need to show that $\left[C,{ }_{s-1} G, C,{ }_{2 i-s} G\right]=$ 1 for every $s=1, \ldots, 2 i$. We use the formula in the proof of 14.5 .4 of [5], which says that if $M, N$ are normal subgroups of a group $G$, then $\left[[M, N],{ }_{n} G\right] \leq$ $\prod_{\ell=0}^{n}\left[\left[M,{ }_{n-\ell} G\right],\left[N,{ }_{\ell} G\right]\right]$. Applying this with $M=\left[C,{ }_{s-1} G\right], N=C$, we have

$$
\left[C,{ }_{s-1} G, C,{ }_{2 i-s} G\right] \leq \prod_{\ell=0}^{2 i-s}\left[\left[C,{ }_{2 i-\ell-1} G\right],\left[C,{ }_{\ell} G\right]\right] .
$$

If $0 \leq \ell \leq i-1$, then $2 i-\ell \geq i+1$, and since $\left[C,{ }_{2 i-\ell-1} G\right] \leq \gamma_{2 i-\ell}(G)$, we have $\left[\left[C,{ }_{2 i-\ell-1} G\right],\left[C,{ }_{\ell} G\right]\right]=1$ by (3). If $\ell \geq i$, then we can argue similarly, since $\left[C,{ }_{\ell} G\right] \leq \gamma_{\ell+1}(G)$.

Theorem 2.6. Let $G$ be a group such that $\left|\gamma_{i+1}(G): \gamma_{i+1}(G) \cap Z_{i}(G)\right|$ is finite. Then $\left|G: Z_{2 i}(G)\right|$ is also finite.

Proof. Let $C_{j}$ be the centralizer in $G$ of $\gamma_{i+j}(G) /\left(\gamma_{i+j}(G) \cap Z_{i-j}(G)\right)$ for $j=1, \ldots, i$. Since $\left|\gamma_{i+j}(G): \gamma_{i+j}(G) \cap Z_{i-j+1}(G)\right|$ is finite by Corollary 2.4 we can apply Proposition 2.3 to the quotient group $G / Z_{i-j}(G)$, and it follows that $\left|G: C_{j}\right|$ is finite. Let $C=\bigcap_{j=1}^{i} C_{j}$, which also has finite index in $G$.

For every $s=1, \ldots, i+1$, put $K_{s}=\left[C,{ }_{s-1} G\right]$, which is contained in $\gamma_{s}(G)$. We prove by reverse induction on $s$ that $K_{s} \cap Z_{2 i-s+1}(G)$ has finite index in $K_{s}$. For 
$s=i+1$ the statement is true, as $\left|K_{i+1}: K_{i+1} \cap Z_{i}(G)\right| \leq\left|\gamma_{i+1}(G): \gamma_{i+1}(G) \cap Z_{i}(G)\right|$ is finite by hypothesis. Now assume that $Z=K_{s+1} \cap Z_{2 i-s}(G)$ has finite index in $K_{s+1}$. As $C$ has finite index in $G$, we have $G=\left\langle g_{1}, \ldots, g_{m}, C\right\rangle$ for some $g_{1}, \ldots, g_{m} \in G$. Let $U=\left\langle g_{1}, \ldots, g_{m}\right\rangle$ and let $H_{s}$ be the centralizer of $U Z / Z$ in $K_{s}$. Since $\left[K_{s}, U\right] Z / Z \leq K_{s+1} / Z$ is finite and $U$ is finitely generated, the subgroup $H_{s}$ has finite index in $K_{s}$. Moreover, $\left[H_{s}, U\right] \leq Z \leq Z_{2 i-s}(G)$ and $\left[H_{s}, C\right] \leq\left[K_{s}, C\right] \leq\left[C,{ }_{s-1} G, C\right] \leq Z_{2 i-s}(G)$, where the last inclusion follows from Lemma 2.5. Hence $\left[H_{s}, G\right]=\left[H_{s}, U C\right] \leq Z_{2 i-s}(G)$ and $H_{s} \leq Z_{2 i-s+1}(G)$, which completes the induction.

In particular, for $s=1$ we obtain that $\left|C: C \cap Z_{2 i}(G)\right|$ is finite. Consequently $\left|G: Z_{2 i}(G)\right| \leq\left|G: C \cap Z_{2 i}(G)\right|=|G: C|\left|C: C \cap Z_{2 i}(G)\right|$ is finite, and we are done.

In order to conclude the proof of Theorem A, it only remains to check that the index $\left|G: Z_{2 i}(G)\right|$ can be actually bounded in terms of $n=\mid \gamma_{i+1}(G)$ : $\gamma_{i+1}(G) \cap Z_{i}(G) \mid$. This depends on the fact that, in the proofs of Proposition 2.3 and Theorem 2.6 every time that we deduce the finiteness of the order or index of a subgroup, we are using one of the following results in a situation where all the invariants involved are bounded in terms of $n$ :

(1) Lemma 2.1.

(2) Lemma 2.2.

(3) Baer's theorem mentioned in the introduction,

(4) Hall's theorem mentioned in the introduction.

For each of these, we have already indicated that there is a quantitative version, and hence Theorem A is now completely proved.

\section{REFERENCES}

1. G. A. Fernández-Alcober and A. Moretó, A finiteness condition on normal subgroups of nilpotent groups, J. Group Theory 5 (2002), 301-315. MR1914347 (2003g:20059)

2. P. Hall, Finite-by-nilpotent groups, Proc. Camb. Philos. Soc. 52 (1956), 611-616. MR0080095 $(18: 190 \mathrm{~d})$

3. I. M. Isaacs, Derived subgroups and centers of capable groups, Proc. Amer. Math. Soc. 129 (2001), 413-416. MR1840087 (2002c:20035)

4. K. Podoski and B. Szegedy, Bounds for the index of the centre in capable groups, Proc. Amer. Math. Soc. 133 (2005), 3441-3445. MR2163577 (2006d:20056)

5. D. J. S. Robinson, A course in the theory of groups. Second edition, Graduate Texts in Mathematics, 80, Springer-Verlag, New York, 1996. MR.1357169 (96f:20001)

6. D. J. S. Robinson, Finiteness conditions and generalized soluble groups. Part 1, SpringerVerlag, New York-Berlin, 1972. MR0332989 (48:11314)

Matematika Saila, Euskal Herriko Unibertsitatea, 48080 Bilbao, Spain

E-mail address: gustavo.fernandez@ehu.es

Dipartimento di Matematica, Università di Bologna, Piazza di Porta San Donato 5, 40127 Bologna, ItALY

E-mail address: mmorigi@dm.unibo.it 